\section{RISCO CARDIOVASCULAR EM MULHERES CLIMATÉRICAS ATENDIDAS EM UNIDADES DE SAÚDE DA FAMÍLIA}

\section{CARDIOVASCULAR RISK IN CLIMATE WOMEN ATTENDED IN PUBLIC HEALTH UNITS}

Daiane dos Santos Souza ${ }^{1}$ / Antônio Nei Santana Gondim ${ }^{1}$ / Darlyane Antunes Macedo ${ }^{1, *}$

\section{INTRODUÇÃO}

A dinâmica do envelhecimento populacional ao longo dos anos e o aumento da expectativa de vida feminina contribui para a possibilidade de mulheres poderem vivenciar o climatério e um período pós- menopausa maior (DIÓGENES; LINARD; TEIXEIRA; 2010).

O climatério é definido como um período de transição entre os anos reprodutivos e não reprodutivos, fazendo parte de um processo fisiológico na vida da mulher. Subsequente ao esgotamento folicular ovariano, e que sobrevém a todas as mulheres, essa fase tem como marco principal a menopausa que condiz ao último ciclo menstrual, sendo identificada de forma retroativa, após 12 meses cessação definitiva dos ciclos menstruais (amenorreia) começando geralmente entre 35 a 40 anos e encerrando até os 65 anos (BRASIL, 2008).

O climatério é tipificado por alterações metabólicas e hormonais que, por vezes, acarreta em mudanças envolvendo aspectos fisiológicos e psicossociais, sendo constantemente associado à sintomatologia característica desse período, como fogachos, alterações de humor, dores de cabeça, suor frio, atrofia urogenital, indisposição, fadiga, zumbidos, vertigem, desconforto musculoesquelético, alterações genitais, insegurança, angústia, justificados pelo estado de diminuição dos níveis de estrogênio, chamado de hipoestrogenismo, que corresponde à queda ou decrescimento significativo de estrogênio (BRASIL, 2008; VERSIANI et al, 2013).

\section{RESUMO}

O climatério é tipificado por alterações metabólicas e hormonais. A diminuição dos hormônios sexuais, que acontece na fase do climatério, pode estar correlacionada com aumento de risco cardiovascular nessas mulheres. O objetivo deste estudo foi comparar o risco cardiovascular das mulheres pré e pós-menopausa assistidas em unidades de saúde da família. Trata-se de um estudo de abordagem quantitativa, descritivo, englobando o total de 50 mulheres de meia idade (26 pré-menopausa e 24 pós- menopausa), atendidas em unidades de saúde da família (USF) do município de GuanambiBahia.Para estimar o risco absoluto para o desenvolvimento de doenças cardiovasculares em 10 anos, foi utilizado o escore de Framinghan para mulheres. Foram definidos como de alto risco cardiovascular escores $>20 \%$; como intermediários entre 10 e $20 \%$; e como baixos quando $<10 \%$. As pacientes pós-menopáusicas apresentam maior risco de eventos cardiovasculares, se comparadas às pré-menopáusicas.

Palavras-chave: Climatério. Menopausa. Risco Cardiovascular.

\section{ABSTRACT}

Climacteric is typified by metabolic and hormonal changes. Decreased sex hormones, which occur in the climacteric phase, may be correlated with increased cardiovascular risk among these women. The aim of this study was to compare the cardiovascular risk of premenopause and postmenopause women assisted in public health units. This is a descriptive and quantitative study involving 50 middle-aged women (26 in premenopause and 24 in postmenopause), attended at public health units in the city of Guanambi-Bahia. To estimate the absolute risk for developing cardiovascular disease in 10 years, the Framinghan score for women was used. Scores $>20 \%$ were defined as high cardiovascular risk; intermediate risk between 10 and 20\%; and low risk when score was $<10 \%$. Postmenopausal patients are at higher risk for cardiovascular events compared to premenopausal patients.

Keywords: Climacteric. Menopause. Cardiovascular risk.

Submetido em: 26 de set. 2019

Aceito em: 28 de mai. 2020

${ }^{1}$ Universidade do Estado da Bahia - UNEB, Guanambi, Bahia - Brasil

*E-mail para correspondência: damacedo@uneb.br

Rev. ComCiência - jun. 2020, vol. 5, no. 6, p. 16-21 / doi: 10.36112/ issn2595-1890.v5.i6.p16-21 
As modificações hormonais, circulatórias e sanguíneas estão reconhecidamente implicadas na gênese e progressão da doença cardiovascular entre essas mulheres. Isso posto, indica-se que o estradiol, um dos três mais importantes estrógenos produzidos no corpo humano, tenha uma atividade protetora no que se refere ao desenvolvimento de doenças cardiovasculares (DCV). Dentre aos meios de cardioproteção, os estrogênios, hormônio sexual feminino produzido pelos ovários e liberado na primeira fase do ciclo menstrual, promovem a redução dos níveis de colesterol total e das lipoproteínas de baixa densidade (LDL), além de aumentarem as lipoproteínas de alta densidade (HDL) (PIAZZA; DE LORENZ; SACILOTO; 2009).

Um dos fatores importantes para o aumento do risco cardiovascular são às alterações metabólicas, como o aumento nos níveis de lipoproteínas de baixa densidade (LDL-colesterol) e a diminuição do efeito protetor de lipoproteínas de alta densidade (HDLcolesterol. Cita-se, ainda, fatores como a hipertensão arterial, de caráter hereditário e associada ao estilo de vida, tais como o tabagismo, diabetes mellitus, obesidade, sedentarismo, alimentação inadequada que induzem a progressão de DCV (BRASIL, 2008).

\section{Com vistas na iminência do} risco cardiovascular aumentando em mulheres climatéricas, é imprescindível que estudos com essa temática sejam produzidos, pois a partir dos resultados encontrados, possíveis planejamentos acerca da atenção a saúde dessas mulheres poderão ser estruturados. O objetivo desse estudo foi comparar o risco cardiovascular das mulheres pré e pós-menopáusicas assistidas em duas unidades de saúde da família na cidade de Guanambi (Bahia) e, assim, considerar possíveis ações para fatores de risco modificáveis, com intuito de estimular mudanças nos hábitos de vida que levem à alteração, em médio e longo prazo, dos fenômenos cardiovasculares dessa população.

\section{METODOLOGIA}

Trata-se de um estudo de abordagem quantitativa, descritivo, englobando 50 mulheres de meia idade, atendidas em duas unidades de saúde da família (USF) do município de Guanambi- Bahia. O referido município localiza-se no sudoeste Baiano, distante 796 km da capital de Salvador.

As USF são localizadas em território urbano, sendo uma das USF situada em bairro periférico e tem um corpo social bastante assíduo e participativo nas atividades propostas pela Unidade. Diferentemente da primeira, a segunda uni dade é localizada no centro, um dos bairros mais antigos do município, com população pouco participativa e com pouca adesão aos serviços oferecidos pelo SUS.

A coleta de dados foi realizada no período de março a abril de 2019 , mediante a utilização de questionário estruturado sobre questões sociodemográficas e clínicas, e do escore de Framingham para mulheres, com mulheres de faixa etária entre 35 e 65 anos, que tivessem exames de lipoproteínas de baixa densidade (LDL-colesterol) e lipoproteínas de alta densidade (HDLcolesterol) realizados nos últimos 6 (seis) meses.

De acordo com o questionário aplicado foi considerada prémenopáusica toda mulher com 35 anos ou mais e ciclos menstruais normal- mente preservados, independentemente de sua regularidade; e foram consideradas pós-menopáusicas aquelas com amenorreia espontânea confirmada por 12 meses ou mais.

Para estimar o risco absoluto para o desenvolvimento de doenças cardiovasculares em 10 anos, foi utilizado o escore de Framinghan para mulheres, é um método que avalia o risco de doença cardiovascular de acordo com a presença ou ausência de alguns fatores de risco, que inclui em seu cálculo, dados referentes à idade, valores séricos de colesterol HDL e LDL, pressão arterial, tabagismo e diabetes. Foram definidos como de alto risco cardiovascular escores $>20 \%$; como intermediários se entre 10 e $20 \%$; e como baixos quando $<10 \%$.

Para avaliar o risco cardiovascular, soma-se as características clinicas de cada paciente, para que medidas de prevenção e recuperação sejam baseadas em fator total e não individual. O Escore de Risco de Framingham - ERF é o indicado pela V Diretrizes Brasileiras sobre Dislipidemias e Prevenção da Aterosclerose, a avaliação de vários fatores de risco ao mesmo tempo permite reconhecer pacientes em alto risco, motiva-las em aderir à terapêutica, associando aos esforços para diminuir os riscos existentes (VERSIANI et al, 2013; MELO et al, 2017).

A análise descritiva dos dados foi realizada por meio de tabelas de frequências simples e tabelas com os valores de médias aritméticas e desvio padrão. O programa Microsoft Excel (Versão 2013) foi utilizado para a análise estatística dos dados. Para fazer as comparações entre os grupos, foram utilizados o teste não paramétrico de Mann-Whitney, para a comparação do fator de risco cardiovascular e o teste 
paramétrico Teste $\mathrm{T}$ de Student para as comparações das demais variáveis quantitativas. Todas as comparações que resultaram em um nível de significância $\mathrm{p}<0,05$ foram consideradas estatisticamente significativas.

Esse estudo obedeceu às normas de pesquisas com seres humanos, de acordo com a resolução n. 466 de 12 de dezembro de 2012, sendo aprovado pelo Comitê de Ética em Pesquisa da Universidade do Estado da Bahia, com o Número do Parecer: 3.068.514

\section{RESULTADOS}

A idade média das mulheres pré-menopáusicas foi de 44,4 \pm 4,7 anos sendo essa variável maior para as mulheres pós-menopáusicas $(57,1 \pm$ 5,2 anos, $\mathrm{p}<0,05)$. A tabela 1 mostra os dados relativos à cor, estado civil e escolaridades das mulheres estudadas.

Tabela 1. Distribuição de frequência relativa à cor, estado civil e escolaridades das mulheres prémenopáusicas e pós-menopáusicas.

\begin{tabular}{llll}
\hline \multirow{2}{*}{ Definição } & $\begin{array}{l}\text { Pré- } \\
\text { menopausa }\end{array}$ & $\begin{array}{l}\text { Pós- } \\
\text { menopausa }\end{array}$ \\
\cline { 3 - 4 } & & $\mathrm{f}(\%)$ & $\mathrm{f}(\%)$ \\
\hline Estado civil & Branca & $7(26,9)$ & $3(12,5)$ \\
& Preta & $2(7,7)$ & $2(8,3)$ \\
& Parda & $16(61,5)$ & $19(79,2)$ \\
& Amarela & $1(3,8)$ & $0(0)$ \\
& Casada & $1(3,8)$ & $2(8,3)$ \\
& Viúva & $17(65,4)$ & $12(50)$ \\
& Divorciadas & $6(23,1)$ & $2(8,3)$ \\
& União estável & $1(3,8)$ & $0(0)$ \\
Escolaridade & Analfabeta & $0(0)$ & $4(16,7)$ \\
& Ensino & & \\
& Fundamental & $11(42,3)$ & $13(24,2)$ \\
& Ensino médio & $12(46,2)$ & $6(25)$ \\
& Ensino superior & $3(11,5)$ & $1(4,1)$ \\
\hline
\end{tabular}

Como pode ser observado na Tabela 2, alguns fatores de risco para doenças cardiovasculares foram estudados. A maior parte das mulheres tanto pré-menopausa como pósmenopausa não são adeptas ao taba- gismo. Em ambos os grupos o Diabetes acomete cerca de $20 \%$ das mulheres. No que diz respeito aos níveis de colesterol LDL, a grande maioria das mulheres pré-menopáusicas $(80,8 \%)$ apresenta níveis abaixo de $150 \mathrm{mg} / \mathrm{dL}$, enquanto que $50 \%$ daquelas pósmenopáusicas possui valores acima de $150 \mathrm{mg} / \mathrm{dL}$. Já para o colesterol HDL ambos os grupos mantiveram, em sua maior parte, valores inferiores a 50 $\mathrm{mg} / \mathrm{dL}$. Valores superiores a 130 $\mathrm{mmHg}$ (PAS) ou $85 \mathrm{mmHg}$ (PAD) foram observados em 53,8 \% das mulheres pré-menopáusicas e $75 \%$ das pós-menopáusicas.

Tabela 2. Distribuição de frequência dos fatores de risco cardiovascular estudado sem mulheres prémenopausa e pós-menopausa.

\begin{tabular}{|c|c|c|c|}
\hline \multirow[t]{2}{*}{$\begin{array}{l}\text { Fatores de } \\
\text { risco } \\
\text { cardiovas- } \\
\text { cular }\end{array}$} & \multirow[t]{2}{*}{ Definição } & $\begin{array}{l}\text { Pré- } \\
\text { menopausa }\end{array}$ & $\begin{array}{l}\text { Pós- } \\
\text { menopau- } \\
\text { sa }\end{array}$ \\
\hline & & $f(\%)$ & f $(\%)$ \\
\hline \multirow[t]{2}{*}{ Tabagismo } & Sim & $0(0)$ & $2(8,3)$ \\
\hline & Não & $26(100)$ & $22(91,7)$ \\
\hline \multirow[t]{2}{*}{ Diabetes } & Sim & $5(19,2)$ & $5(20,8)$ \\
\hline & Não & $21(80,8)$ & $19(79,2)$ \\
\hline \multirow{2}{*}{$\begin{array}{l}\text { Colesterol } \\
\text { LDL }\end{array}$} & $<150 \mathrm{mg} / \mathrm{dL}$ & $21(80,8)$ & $12(50)$ \\
\hline & $\geq 150 \mathrm{mg} / \mathrm{dL}$ & $5(19,2)$ & $12(50)$ \\
\hline \multirow{2}{*}{$\begin{array}{l}\text { Colesterol } \\
\text { HDL }\end{array}$} & $<50 \mathrm{mg} / \mathrm{dL}$ & $23(88,5)$ & $17(70,8)$ \\
\hline & $\geq 50 \mathrm{mg} / \mathrm{dL}$ & $3(11,5)$ & $7(29,2)$ \\
\hline \multirow[t]{2}{*}{$\begin{array}{l}\text { Pressão } \\
\text { arterial }\end{array}$} & $\begin{array}{l}\mathrm{PAS} \text { ou } \leq 85 \\
\mathrm{mmHg} \mathrm{PAD}\end{array}$ & $12(46,2)$ & $6(25)$ \\
\hline & $\begin{array}{l}\geq 130 \mathrm{mmHg} \text { PAS } \\
\text { ou }>85 \mathrm{mmHg} \\
\text { PAD }\end{array}$ & $14(53,8)$ & $18(75)$ \\
\hline
\end{tabular}

A Tabela 3 mostra a média e o desvio padrão dos fatores de risco de doenças cardiovasculares. Nela pode ser observado que não houve diferença estatisticamente significativa quanto a PAS e PAD quando comparados os grupos de mulheres pré e pósmenopáusicas. Todavia ao se analisar os dados de colesterol, tanto HDL quanto LDL, esses parâmetros mostraram-se maiores no grupo de mulheres pós-menopáusicas ( $\mathrm{p}<0,05)$.
Tabela 3. Média aritmética dos fatores de risco cardiovascular em mulheres pré-menopausa e pósmenopausa

\begin{tabular}{|c|c|c|c|}
\hline \multirow{2}{*}{$\begin{array}{l}\text { Fatores de } \\
\text { risco cardio- } \\
\text { vascular }\end{array}$} & $\begin{array}{l}\text { Pré- } \\
\text { menopausa }\end{array}$ & \multicolumn{2}{|l|}{$\begin{array}{l}\text { Pós- } \\
\text { menopausa }\end{array}$} \\
\hline & $\begin{array}{l}\text { Média } \\
\pm \text { Desv.pad. }\end{array}$ & $\begin{array}{l}\text { Média } \\
\pm \text { Desv.pad. }\end{array}$ & $\begin{array}{l}\text { Testet de } \\
\text { Student }\end{array}$ \\
\hline Colesterol & 102,9 & 153,7 & $\mathrm{p}$ \\
\hline LDL & 43,7 & 41,5 & $0,00011^{*}$ \\
\hline $\begin{array}{l}\text { Colesterol } \\
\text { HDL }\end{array}$ & $39,1 \pm 8,7$ & $46,5 \pm 13$ & $\mathrm{p}_{0,022 *}=$ \\
\hline PAS (mmHg) & $132 \pm 23,4$ & $\begin{array}{l}131,3 \quad \pm \\
12,5\end{array}$ & $\mathrm{p}=0,896$ \\
\hline $\mathrm{PAD}(\mathrm{mmHg})$ & $85,8 \pm 12,4$ & $83,7 \pm 9,7$ & $\mathrm{p}=0,520$ \\
\hline
\end{tabular}

*p<0,05: nível de significância estatística, PAS = pressão arterial sistólica, PAD = pressão arterial diastólica, Desv.Pad = desvio padrão.

O risco cardiovascular calculado para os próximos 10 anos está mostrado na Tabela 4. Nela é possível observar que 83,3\% nas mulheres pósmenopáusicas possuem risco intermediário e alto. Esse valor cai para 30,7 \% no grupo pré-menopáusico.

Tabela 4. Distribuição de frequência de risco cardiovascular em mulheres pré-menopausa e pósmenopausa

\begin{tabular}{lll}
\hline Risco cardiovas- & Pré-menopausa & Pós-menopausa \\
\cline { 2 - 3 } cular & $\mathrm{f}(\%)$ & $\mathrm{f}(\%)$ \\
\hline Baixo & $18(69,3)$ & $4(16,7)$ \\
Intermediário & $3(11,5)$ & $14(58,3)$ \\
Alto & $5(19,2)$ & $6(25)$ \\
Total & $26(100)$ & $24(100)$ \\
\hline \multicolumn{2}{c}{ f: Frequência observada }
\end{tabular}

Quando comparado os dois grupos, quanto ao risco cardiovascular, foi observado que as mulheres prémenopáusicas apresentaram uma média de $9,8 \pm 11 \%$, valor que foi aumentado para $17,5 \pm 8,7 \%(\mathrm{p}<0,05)$ o que mostra que a pós-menopausa de fato aumenta o risco de doenças cardiovasculares (ver tabela 5).

Tabela 5. Média aritmética do risco cardiovascular em mulheres pré-menopausa e pós-menopausa.

\begin{tabular}{|c|c|c|}
\hline Risco cardio- & $\begin{array}{l}\text { Pré- } \\
\text { menopausa }\end{array}$ & $\begin{array}{l}\text { Pós- } \\
\text { menopausa }\end{array}$ \\
\hline vascular & $\begin{array}{l}\text { Média } \\
\pm \text { Desv.pad. }\end{array}$ & $\begin{array}{ll}\text { Média } & \text { Teste de Man- } \\
\pm \text { Desv.pad. } & \text { Whitney }\end{array}$ \\
\hline & $9,8 \pm 11 \%$ & $17,5 \pm 8,7 \% \quad \mathrm{P}<0,0001 *$ \\
\hline
\end{tabular}




\section{DISCUSSÃO}

A literatura tem evidenciado o climatério, em especial o período pósmenopausa, o de maior risco cardiovascular para a mulher, observa-se que as mulheres passam por um processo gradativo de mudanças fisiológicas, certamente em consequência da redução do estrogênio, o que resulta em mudanças mais prolongadas, no período pós-menopausa, essa mudança hormonal indica ter efeito direto sobre eventos cardiovasculares (PIAZZA; DE LORENZI; SACILOTO 2009; MELO et al, 2017).

No que se refere ao climatério, a maior parte das mulheres desse estudo se encontrava no período prémenopausa, estando 26 na prémenopausa e 24 pós-menopausa. A idade média das mulheres prémenopáusicas foi de 44,4 \pm 4,7 anos sendo essa variável maior para as mulheres pós-menopáusicas $(57,1 \pm 5,2$ anos, $\mathrm{p}<0,05)$. Ressaltando a relevância do conhecimento da idade de ocorrência dos episódios de climatério e menopausa nos diferentes públicos.

A maioria das mulheres era de cor parda, seguida por branca e preta. Coincidindo com as características constatada por Veloso et al. (2014) Mulheres do grupo pré-menopausa apresentaram valores para as seguintes etnias: Parda 61,5\%, Brancas 26,9\%, preta $7,7 \%$, sendo as pós- menopausa Pardas 79,2\% Brancas 12,5\% Pretas $8,3 \%$. Contudo o estudo não realizou correlação com DCV.

Neste trabalho notou-se que as mulheres do grupo pós-menopausa tem níveis escolaridade inferior ao grupo de pré- menopausa. Similar aos achados de Veloso et al.(2014); fundamentando a conclusão de que quanto menos anos de estudo, mais dificuldade em relação aos cuidados preventivos para qualquer doença. Na maioria das vezes ao se aproximarem da menopausa, as mulheres trazem anseios, dúvidas e apreensões sobre as características do período e as mudanças previstas. Logo, é de extrema relevância ações de educação em saúde voltadas para a temática, pois, à medida que as mesmas esclarecem suas dúvidas em relação ao climatério, não só restringem sua ansiedade, mas também tem a possibilidade de adotarem um estilo de vida mais saudável, o que melhora a prevenção de futuras complicações e qualidade de vida. (PIAZZA, DE LORENZI, SACILOTO, 2009).

O tabagismo é considerado um fator de risco de destaque para doença cardiovascular; esse causa o aumento dos níveis de LDL-colesterol e da pressão arterial. No presente estudo a maioria das mulheres, tanto as prémenopausa como pós-menopausa não são adeptas ao tabagismo, um coeficiente positivo, no que diz respeito às doenças cardiovasculares, pois o tabagismo é um fator de risco e o seu combate deve fazer parte das estratégias de ação dos serviços de saúde. Esses achados diferem-se do estudo em que participaram 164 mulheres, dentre as mulheres presentes na pesquisa, $24 \%$ das pré-menopáusicas e $12,3 \%$ das pós-menopáusicas faziam uso do tabaco, o que, comprovadamente, leva a um aumento no risco de DCV (VERSIANI et al., 2013).

Em ambos os grupos, pré e pósmenopausa o Diabetes acomete cerca de $20 \%$ das mulheres. A doença cardiovascular é a prevalecente na causa de morbimortalidade em pessoas com diabetes mellitus $\mathrm{O}$ diabetes mellitus e as doenças cardiovasculares estão intimamente relacionadas sendo uma das principais causas de DCV (LARRÉ, ALMEIDA, 2014).
Em relação aos níveis de LDLcolesterol, a grande maioria das mulheres pré-menopáusicas (80,8 \%), apresenta níveis abaixo de $150 \mathrm{mg} / \mathrm{dL}$, enquanto que $50 \%$ daquelas pósmenopáusicas possui valores acima de $150 \mathrm{mg} / \mathrm{dL}$. O estado de hipoestrogenismo, resultante do esgotamento folicular ovariano, faz com que os níveis de LDL-colesterol tendem a aumentar, contribuindo com um dos fatores de risco para doença cardiovascular.

Quanto aos níveis HDLcolesterol, ficaram alterados entre as mulheres climatéricas pré e pós- menopausa, os valores estavam abaixo de $50 \mathrm{mg} / \mathrm{dL}$, indicando que o efeito protetor de HDL-colesterol pode estar diminuído em mulheres em transição da menopausa. Esses resultados corroboram com o estudo de Melo et al. (2017), onde foram encontrados valores baixos de HDL-colesterol em mulheres da mesma faixa etária.

No que diz respeito aos valores de pressão arterial sistólica e diastólica foram observados $53,8 \%$ das mulheres pré-menopáusicas e $75 \%$ das pósmenopáusicas com valores superiores a $130 \mathrm{mmHg}$ (PAS) ou $85 \mathrm{mmHg}$ (PAD). A hipertensão arterial sistêmica aumenta progressivamente com a idade, esse processo acontece nas mulheres principalmente no início da fase pós-menopausa. De acordo com estudos, durante a pré-menopausa, os níveis pressóricos femininos tendem a ser inferiores o que tem sido atribuído à ação dos estrógenos endógenos. No entanto, após a menopausa, os níveis pressóricos da mulher tendem a se elevar significativamente (MELO, 2017).

Através do escore de risco de Framingham avaliou-se o risco cardiovascular da amostra, podendo notar que 83,3 \% nas mulheres pósmenopáusicas possuem risco interme- 
diário e alto, comparadas a 30,7 \% no grupo pré-menopáusico. Consolidando-se com o trabalho de Piazza et al. (2009). Demostrando a associação relevante entre o estado pósmenopausa e os escores de risco cardiovascular proposto por Framinghan.

Foi observado que as mulheres pré-menopáusicas apresentaram uma média de 9,8 $\pm 11 \%$ do risco para doença cardiovascular, valor que foi aumentado para $17,5 \pm 8,7 \%$ nas mulheres que se encontram na pósmenopausa. $\mathrm{O}$ que mostra que a pósmenopausa de fato aumenta o risco de doenças cardiovasculares. De acordo com Dallazen et al. (2017) onde seus resultados mostram que as mulheres no período Pós- menopausa apresentavam valores superiores, expondo desenvolvimento de risco para doença cardiovascular, mais graves no período pós menopausa.

\section{CONCLUSÃO}

Conclui-se que as mulheres pósmenopáusicas apresentam maior risco de desenvolvimento de doenças cardiovasculares, se comparadas às prémenopáusicas. Assim, faz-se necessária a ampliação da assistência direcionada a essas pacientes na prevenção primária de saúde, com o intuito de reduzir a morbimortalidade dos eventos cardiovasculares dessa população. O reconhecimento antecipado dos agravos acometidos durante o climatério e dos fatores de risco para desenvolver doenças cardiovasculares em mulheres climatéricas auxilia na redução de mortalidade desse grupo.

A fase climatério e menopausa como já citado, é um período de ambiguidades para muitas mulheres, o que pode refletir negativamente na sua saúde. Esse estudo sugere que mulheres mesmo na pré-menopausa apresen- taram níveis baixos de HLD, Salientando a importância da assistência apropriada ainda na fase inicial do climatério, sendo indispensável para que possa diminuir as repercussões e abalos gerados no processo de mudanças físicas e psicológicas que comumente acontece durante o climatério.

Os fatores de risco para doença cardiovascular incluem hábitos modificáveis, relacionados ao estilo de vida, o controle e mudança desses fatores, compreendidos como alimentação adequada, atividade física, controle da pressão arterial e taxas de colesterol, evitar o tabagismo e álcool, são fundamentais na prevenção de eventos cardiovasculares em médio e longo prazo.

O predomino de fatores de risco modificáveis para doenças cardiovasculares corrobora com a seriedade e o papel das políticas públicas de saúde voltadas à atenção primária à saúde da mulher no seu processo de envelhecimento. Espera-se que os resultados obtidos possam contribuir na prevenção de agravos e mudanças dos fatores de risco para o desenvolvimento de doenças cardiovascular voltadas a esse público.

\section{REFERÊNCIAS}

BRASIL. Ministério Da Saúde. Secretaria De Atenção À Saúde. Departamento De Ações Programáticas Estratégicas. Política nacional de atenção integral à saúde da mulher: princípios e diretrizes. Ministério da Saúde, 2008.

BRASIL. Ministério Da Saúde. Manual de atenção à mulher no climatério/menopausa. Ministério da Saúde, 2004.
DALLAZEN, Fernanda; WINKELMAN, Eliane; BERLEZI, Evelise Moraes. Risco cardiovascular avaliado pelo índice de conicidade em mulheres no climatério: análise comparativa entre os períodos pré e pósmenopausa. Scientia Medica, v. 27, n. 4, p. 3, 2017.

\section{DIÓGENES, Maria Albertina}

Rocha; LINARD, Andrea Gomes; TEIXEIRA, Carla Araujo Bastos. Comunicação, acolhimento e educação em saúde na consulta de enfermagem em ginecologia. Revista da Rede de Enfermagem do Nordeste, v. 11, n. 4, p. 38-46, 2010.

\section{LARRÉ, Mariana Costa; AL-} MEIDA, Elayne Conceição de Souza. Escore de Framingham na avaliação do risco cardiovascular em diabéticos. Revista da Rede de Enfermagem do Nordeste, v. 15, n. 6, 2014.

MELO, Jorgileia Braga de et al. Fatores de risco cardiovascular em mulheres climatéricas com doença arterial coronariana. Int. j. cardiovasc. sci. v. 31 , n. 1, p. f: 4-1: 11, 2017.

PIAZZA, Ivanete Perboni; DE LORENZI, Dino Roberto Soares; SACILOTO, Bruno. Avaliação do risco cardiovascular entre mulheres climatéricas atendidas em um programa de saúde da família. Revista Gaúcha de Enfermagem, v. 26, n. 2, p. 200, 2009.

Versiani CM, Freire AC, Dias GMM, Brito BD, Rocha JSB, Reis VMCP. Avaliação do risco cardiovascular em mulheres climatéricas assistidas pelo Programa Saúde da Família. Revista da 


\section{Sociedade Brasileira de Clínica}

Médica. 2013 out-dez;11(4).

VELOSO, Gilson Gabriel Viana

et al. Prevalência de síndrome

metabólica em mulheres climaté-

ricas. RevBrasCardiol, v. 27, n.

1, p. 20-27, 2014. 